\title{
Skin inflammation is associated with matrix remodelling and MMP expression and
}

activation in hidradenitis suppurativa

Julia SANCHEZ ${ }^{1,2}$, Sébastien LE JAN ${ }^{1}$, Céline MULLER ${ }^{1}$, Caroline FRANCOIS ${ }^{3}$, Yohan RENARD ${ }^{4}$, Anne DURLACH ${ }^{3}$, Philippe BERNARD ${ }^{1,2}$,

$\{2\}$ Ziad REGUIAI ${ }^{2}$, Frank ANTONICELLI ${ }^{1}$

\begin{tabular}{l}
${ }_{5}^{5}$ Laboratory Pol Bouin, Maison Blanche, Reims University Hospital, Reims, France \\
\hline
\end{tabular}

\section{INTRODUCTION}

BACKGROUND

(dill unknown. (1) metalloproteinase (MMP)-2 were detected both in the perilesional and in the uninvolved HS skin. Interestingly, MMP expression is induced by many pro-inflammatory cytokines. An inflammasome has been identified in HS keratinocytes, resulting production of IL-1 $1 \beta$. Besides, it has been proposed that the production of IL-17 by neutrophils in the deep dermis could lead to the activation of the inflammasome. However, there stil is a missing link proving that production of IL-17 at distance from keratinocyte could participate to an auto amplification loop through the recruitment and activation of inflammatory factors. We here wondered whether skin structural alteration could participate to disease progression.

\section{OBJECTIVE}

To factors present in involved versus non-involved HS skin and in control skin on skin structural parameters such as collagen fibres content and spatial organisation, and on MMP-2 and MMP-9 expression. We also sought a link between the expressions of the cytokines IL-1 $\beta$ and IL-17 an those of the inflammasome-associated proteins NLRP3 and caspase-1.

\section{METHODS}

METHODS

Skin biopsies were harvested from surgery skin explants from obese patients or patients with HS (lesional and peri-lesional) and were cultured. Both at day 0 and day 4 , one set of skin samples was fixed, embedded in paraffin and sectioned to perform tissue morphology (Haematoxylin/eosin staining), matrix remodeling (collagens and elastin were analyzed by second harmonic generation (SHG) and autofluorescence), and inflammasome activation analysis (Immunostaining with a monoclonal anti-human NLRP3 antibody (Enzo Life Science, Inc.) or a polyclonal anti-human Caspase-1 antibody (Cell Signaling Technology, Inc.)). At the same time points, a second set of skin explants was used to prepare skin homogenate extracts in order to determine cytokine concentration (IL-17 and IL-1 $\beta$, ELISA assay, BioTechne and Ebiosciences respectively), MMP-2 and MMP-9 levels (gelatin zymography), and to stimulate keratinocytes HaCat.

\section{STATISTICAL ANALYSES}

tatistics, such as means, SDs, or SEMs were conducted for all quantitative measures. Because of the quite small number of cases in this study, we used nonparametric testing to compare populations

(Mann-Whitney test) and to determine correlation coefficient (Spearman's correlation coefficient). The results were considered significant if $P$ values were .05 or less.

\section{RESULTS (2)}

Inflammatory cytokines and inflammasome in HS lesional and perilesional skin in culture

(A)
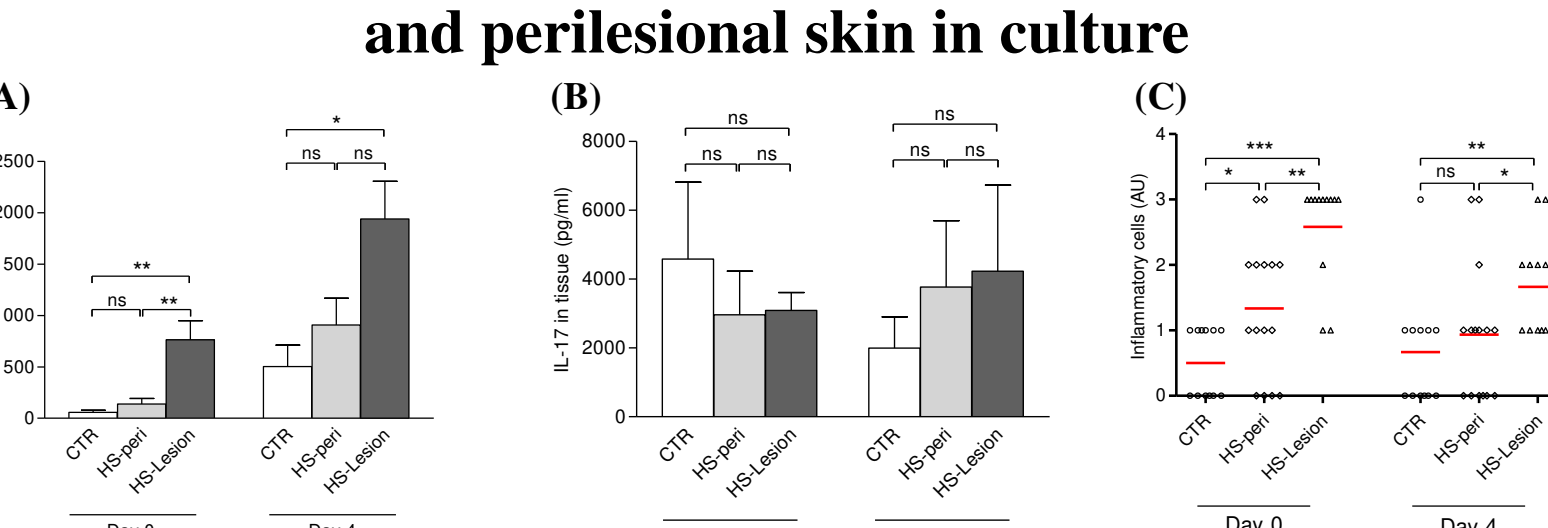

Figure 2: Elevated and increased IL-1 $\beta$ concentration in HS skin explants while IL 17 concentration is not correlated with inflammatory cells. IL-1 $\beta$ (A) and IL-17 (B) concentrations were measured by ELISA in homogenates of perilesional or lesional skin explants at day 0 and after 4 days of culture. The number of inflammatory infiltrated cells in HS perilesional and lesional skin explants was evaluated from HE stained skin sections by 2 independent investigators who assigned a number between 0 and 4 to notify the absence (0) or presence of inflammatory cells (1: very low; 2 : low; 3 : moderate and 4: high) (A)
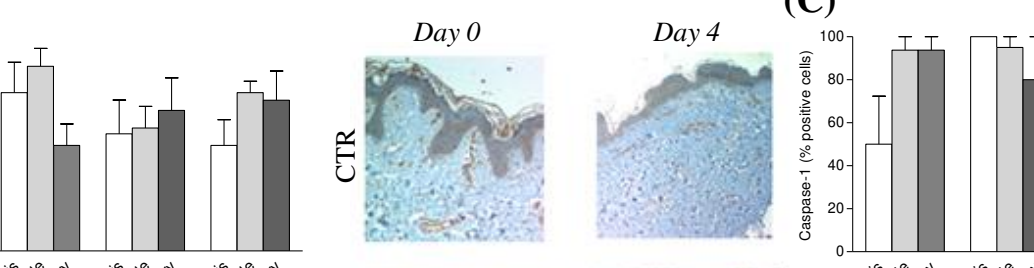

(B)

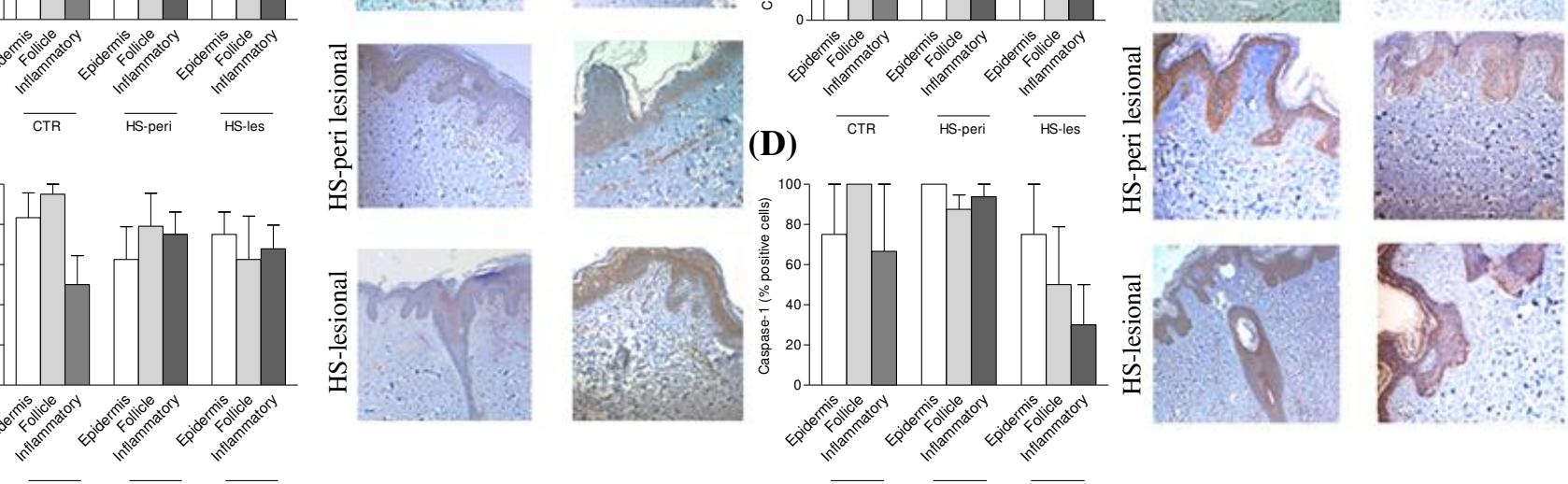

Figure 3: Presence of inflammasome in HS and control skin, without variation during culture. performed perilesional and lesional skin explants from HS at day 0 (A-C) or after 4 days of culture (B-D), b independent investigators who evaluated the percentage of positive cells.
ind Caspase 1

\section{ACKNOWLEDGEMENT}

Société de Recherche en Dermatologie (SRD) and Société Française de Dermatologie (SFD) for their financial participation.

Plateforme IbiSA "Imagerie Cellulaire et tissulaire" for providing microscopy equipment

\section{PATIENTS}

Nine consecutive patients with HS, classified according to the Hurley staging and nine obese patients that underwent dietary and skin tissue reduction considered as control patients were enrolled in the department of dermatology, and in the department of visceral surgery, and the department of plastic surgery at the university hospital of Reims, France, respectively. The study was approved by the Ethic Committee of the University Hospital of Reims (CPP 23.01.2015), and all of the subjects gave their informed and written consent before participating in the study in accordance with the Helsinki Declaration.

\begin{tabular}{|c|c|c|c|c|c|c|c|c|}
\hline & Age & & & Aurle & & Topogr & y of sk & amples \\
\hline & $\begin{array}{c}\text { (median } \\
\text { уо) }\end{array}$ & Gender & 1 & $\|$ & III & Armpits & Pubis & $\begin{array}{c}\text { Lower } \\
\text { back }\end{array}$ \\
\hline $\begin{array}{l}\text { Control } \\
\text { patients }\end{array}$ & 41 & $7 F / 2 M$ & & NA & & 2 & 4 & 3 \\
\hline $\begin{array}{c}\text { HS } \\
\text { patients }\end{array}$ & 31 & $7 F / 2 M$ & 1 & 5 & 3 & 9 & 0 & 0 \\
\hline
\end{tabular}
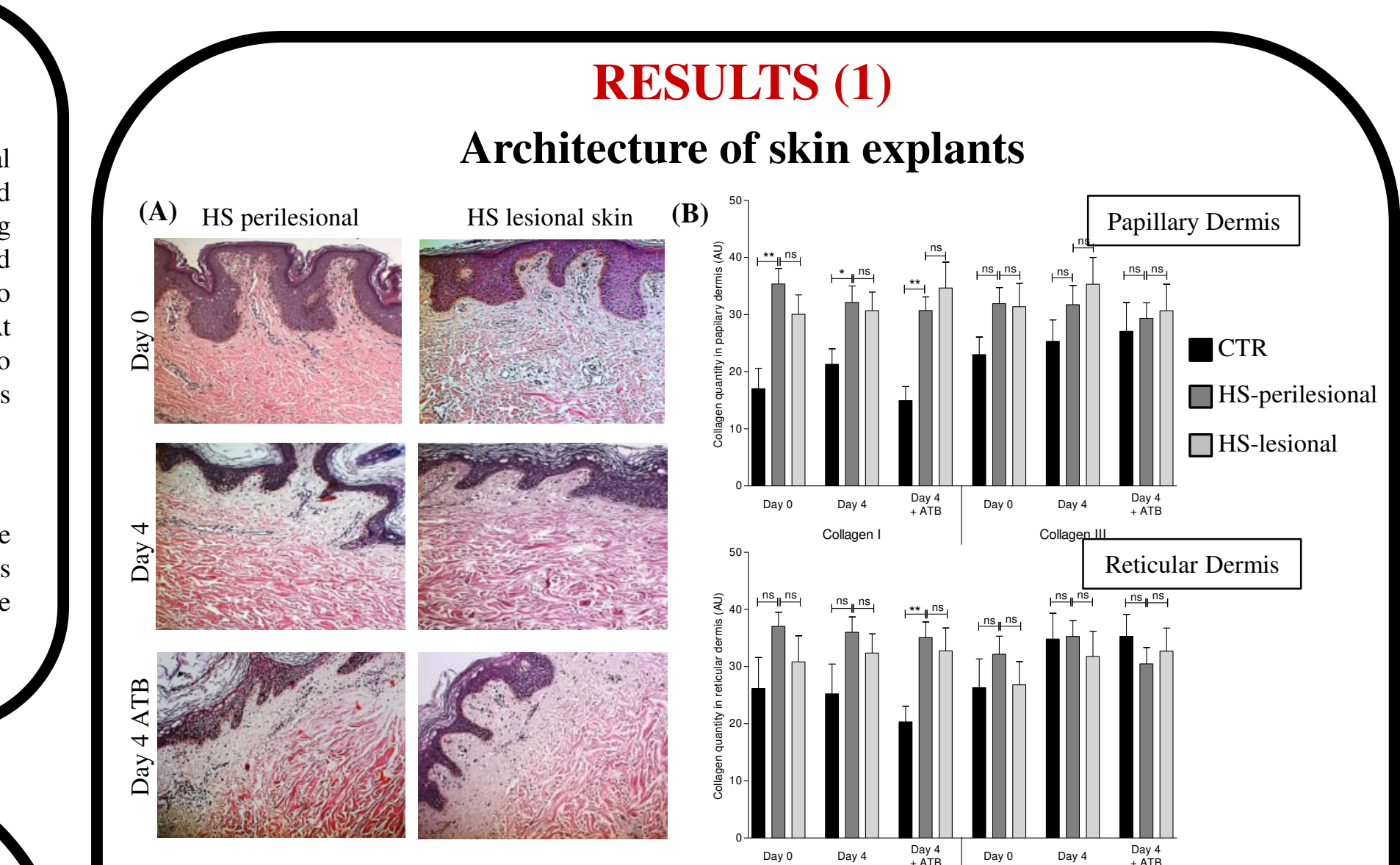

Figure 1: Microscopic architecture of skin explants is conserved during culture, associated with an increase of collagen I level in papillary dermis of HS skin. (A) Haematoxylin/eosin staining of perilesional and lesional skin explants from HS maintained in culture during 4 days with (Day 4 ATB) or without (Day 4) Rifampicin and Clindamycin. (B) Quantification of collagen fibers in papillary and reticular dermis of skin explants by computer-assisted digital image analysis of picrosirius stained skin sections.

RESULTS (3)

Alteration of fibrillar network in HS skin

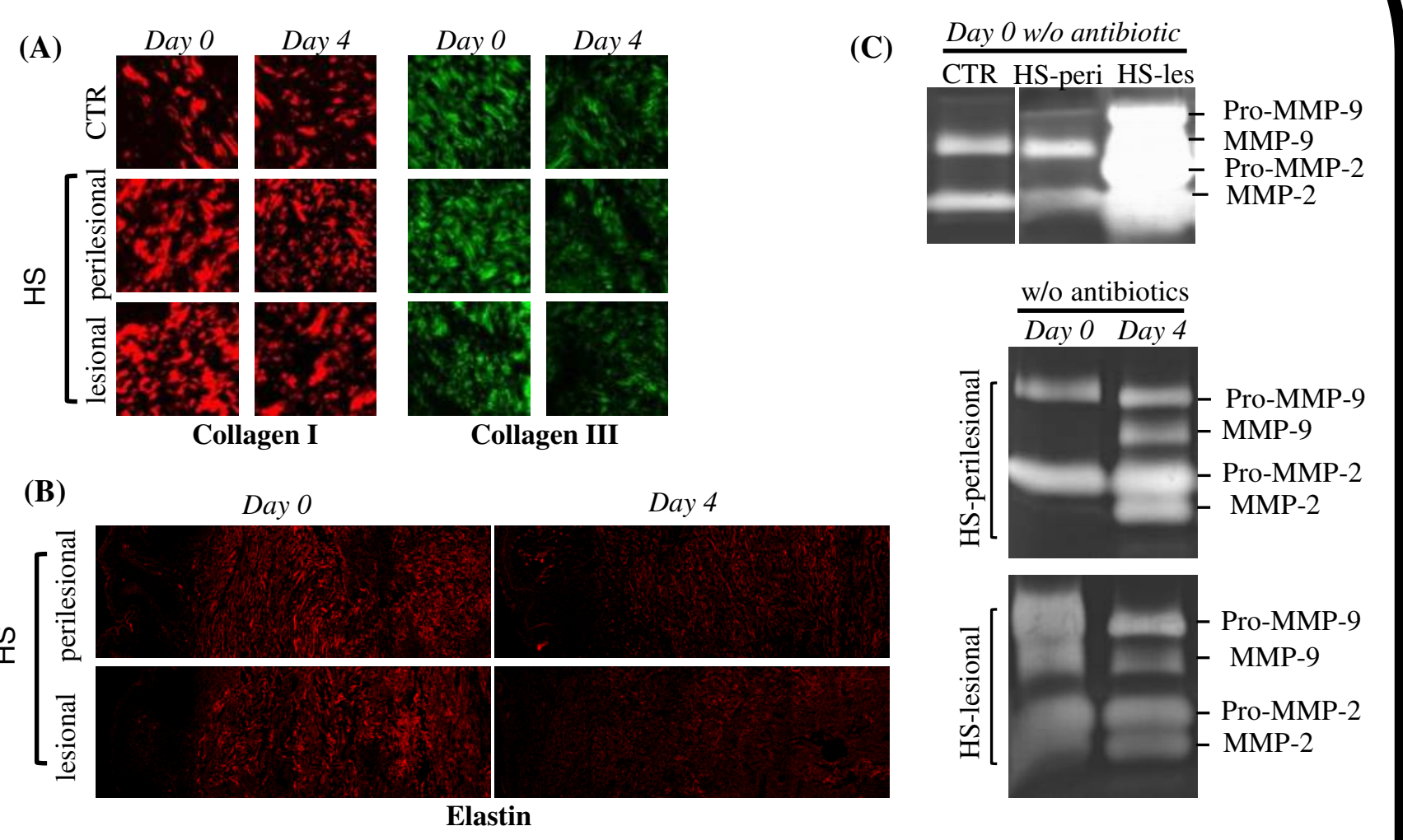

Figure 4: Alteration of collagen and elastin network in relation with MMP secretion. Collagen (A) and elastin (B) structure evaluated respectively by computer-assisted digital image analysis of picrosirius stained skin sections and second harmonic generation. (C) Evaluation by gel zymography lesional skin explants at day 0 or cultured ex vivo for 4 days. 